\title{
Numerical Modeling on Anisotropy of Seepage and Stress Fields of Stratified Rock Slope
}

\author{
Honglei Liu, ${ }^{1}$ Wenhao Shi $\mathbb{D},^{2}$ and Tianhong Yang $\mathbb{D}^{1}$ \\ ${ }^{1}$ School of Resources and Civil Engineering, Northeastern University, Shenyang 110819, China \\ ${ }^{2}$ School of Civil Engineering, Suzhou University of Science and Technology, Suzhou 215011, China \\ Correspondence should be addressed to Wenhao Shi; shiwenhaoneu@126.com
}

Received 3 June 2019; Accepted 10 March 2020; Published 7 April 2020

Academic Editor: Fabrizio Greco

Copyright (c) 2020 Honglei Liu et al. This is an open access article distributed under the Creative Commons Attribution License, which permits unrestricted use, distribution, and reproduction in any medium, provided the original work is properly cited.

Stratified rock mass is a common rock mass structure and distributed widely on the earth's crust. High-density distribution of stratified structural planes in the stratified rock mass has a great influence on the mechanics properties of the rock mass, such as anisotropy of stress and seepage. Therefore, anisotropy is the inherent characteristic that must be considered in the stratified rock mass as well as the stratified rock slope stability. According to the anisotropic characteristics of stratified rock mass, an anisotropic seepage-stress coupled model for the mechanical behavior of stratified rock slope is proposed based on the anisotropy elasticity theory. The model is validated by simulating seepage and stress fields of an idealized slope using the finite element method. The deformation and stress-dependent permeability of the slope are predicted and compared well with the previous study, thus confirming its capability in characterizing the response of rock slope that is dominantly affected by rock anisotropy. Finally, the model is used to simulate the anisotropic properties of a stratified rock slope of Fushun west open-pit mine, China. The simulation is in good agreement with the actual measurement, which means the proposed model is capable of simulating anisotropic properties of the stratified rock slope.

\section{Introduction}

Open-pit mining is the most important technology for extracting mineral resources from the earth's crust. Production rates of open pit have progressively grown over the last 100 years and it will continue to grow in the future [1]. In open-pit mines, the economic benefits and total minable reserve are severely influenced by rock slope stability which is affected by different uncertainties arising from many sources. The slope rock mass that contains multiple structural planes has a certain structure, such as stratified structure, which is very common and distributed widely in the earth's crust. These stratified structural planes are primordially distributed in stratified rock mass. When such rock strata are excavated by open-pit mining, the stability of stratified rock slope will be an inevitable problem for the engineering. The stratified structural plane not only leads to the heterogeneity and anisotropy of rock mass but also affects the failure mode of rock slope. Therefore, it is of great significance to characterize these structural planes and quantify their effect on the mechanical response of rock slope in order to evaluate the rock slope stability.

Rock slope stability assessment based on the rock mass classification system is an effective method for the general understanding of the landslide-prone slope. Extensive efforts on this method have been made to assess rock slope stability. The existing slope stability assessment methods summarized by Pantelidis [2] as well as probabilistic analysis methods $[3,4]$ are helpful for the more-refined and quantitative analysis of the rock slope stability in specific areas.

In practice, the traditional limit equilibrium method with less parameter involved is widely used for slope stability analysis, such as the Fellenius method, Bishop method, Janbu method, and Sarma method. All these methods are proposed based on the principle of statical equilibrium and have been well summarized by Duncan [5]. However, in these methods, the stress and deformation properties cannot be obtained due to the rigid assumption of landslide mass 
which is not accurate for the stress-controlled landslide [6]. In this respect, the finite element method (FEM) which makes up the insufficiency has become popular for slope stability analysis due to its simplicity and feasibility. Ma et al. [7] simulated the failure mode of slopes with different dip angles using finite element programming and found that the critical failure surface develops along the weak structural surface when the slope dip angle is below $30^{\circ}$. Zhang et al. [8] calculated the stress and displacement fields of a layered slope during the drawdown of water level using FEM and compared the results with the experimental observation and measurement. Some researchers introduced the shearstrength reduction technique into FEM for the slope stability analysis [9]. In these studies, the rock mass is still treated as an isotropic medium which may be not suitable and acceptable for the stratified rock slope because the stratified rock mass usually be characterized as the strongly anisotropic material [10].

In order to characterize the structural planes in rock mass, many models are proposed, which can be classified as continuum mechanical model and discontinuous mechanical model. The former model is established based on the proper rock mechanics model to study macroscopic deformation, seepage, and other mechanical response of the rock mass. For a large-scale structural plane such as regional faults, it can be considered as an independent domain by specifying the appropriate parameters for rock mechanics analysis. The discontinuous deformation analysis (DDA) proposed by Shi [11] is internationally recognized in effectively quantifying the response of every structural planes in the rock mass, obtaining a good application in practical engineering. However, as for the huge number of stratified structural planes with very small spacing, it is really difficult to explicitly represent all the structural planes in DDA modeling. Even worse, it lacks practical significance and possibility for fully considering the structural planes at each scale. Therefore, the rock mass under certain conditions can be considered as a continuum medium, and its behavior could also be studied using the continuum mechanical model [12]. In this respect, during the seepage analysis of rock mass, the representative elementary volume may exist, and the equivalent continuum model could be used when the size of representative elementary volume (REV) varies between $1 / 50$ and $1 / 20$ of the engineering dimension [13].

Anisotropy is the inherent characteristic that must be considered in rock engineering analysis. Amadei [14] pointed out many rocks near the earth's surface that show well-defined fabrics in the form of bedding, stratification, layering, foliation, fissuring, or jointing. These rocks can then have properties (e.g., physical, thermal, mechanical, and hydraulic) which vary in different directions inducing anisotropic behavior. Experimental investigations reveal that rocks are anisotropic in deformation, seepage, and strength. When the equivalent continuum approach is used for regularly fractured rock masses, anisotropy must be considered because it can be quite significant because of the major deformations along the discontinuities $[15,16]$. Due to the variety of rock properties with anisotropic behavior, neglecting the effect of anisotropy may increase the error in stress measurement [17], ground control [18], and damage estimation during underground construction $[19,20]$.

In this study, an anisotropic seepage-stress coupled model is proposed to characterize the anisotropy and seepage-stress coupled properties of the stratified rock mass at macro level. The Louis empirical formula is generalized to describe the anisotropic problem of the stratified rock mass. The model is validated by simulating the stress and seepage fields of an idealized slope using the finite element method. In order to further verify the engineering applicability of the proposed model, a stratified rock slope of Fushun west openpit mine is simulated using the model. The simulations are in good agreement with the actual measurements. This study may provide reference for more accurate analysis of the mechanics behavior of the stratified rock slope.

\section{Governing Equations of the Numerical Model}

Description of an anisotropic rock mass in math is the precondition for the corresponding mechanical properties by numerical simulation. Once the constitutive model is defined, more efficient continuum numerical analyses based on the equivalent properties can be adopted. In order to build the constitutive model, two assumptions should be confirmed initially: (i) anisotropy of the stratified rock mass is predominantly controlled by the stratified structural planes; (ii) stratified rock mass can be treated as an elastic, homogeneous, and anisotropic medium.

2.1. Governing Equations for Anisotropic Stress Field. As per the above assumptions, if a REV is extracted from the stratified rock slope, the REV can be treated as an anisotropic and elastic medium. Then, the equilibrium equation of the REV under the plane strain condition can be expressed as

$$
\sigma_{i j, j}+F_{i}=0, \quad i, j=1,2,
$$

where $\sigma_{i j}$ is the total stress tensor and $F_{i}$ is the body force.

Based on generalized Hook's law, the constitutive relation without groundwater seepage can be expressed as

$$
\sigma_{i j}=[D] \varepsilon_{i j},
$$

where $\varepsilon_{i j}$ is the strain tensor which can be calculated by the displacement and $[D]$ is the elastic matrix which can be expressed in the following form:

$$
\begin{aligned}
{[D] } & =\left[\begin{array}{ccc}
\frac{E_{1}-E_{2} v_{2}^{2}}{E_{2} \Delta} & \frac{\left(1+v_{1}\right) v_{2}}{\Delta} & 0 \\
\frac{\left(1+v_{1}\right) v_{2}}{\Delta} & \frac{1-v_{1}^{2}}{\Delta} & 0 \\
0 & 0 & G_{12}
\end{array}\right], \\
\Delta & =\frac{E_{1}-E_{2} v_{1} v_{2}-E_{1} v_{1}^{2}-E_{2} v_{2}^{2}-2 E_{2} v_{1}^{2} v_{2}}{E_{1} E_{2}},
\end{aligned}
$$


where $E_{1}$ and $E_{2}$ are Young's modulus parallel and perpendicular to the stratified structural planes, respectively; $v_{1}$ and $v_{2}$ are Poisson' ratio parallel and perpendicular to the stratified structural planes, respectively; and $G_{12}$ is the shear modulus in the direction parallel to the stratified structural planes.

In order to describe the seepage effect on the stress field, a classical relation called effective stress principle was proposed by Terzaghi et al. [21]. The effective stress, $\sigma_{i f}^{\prime}$ can be expressed by the total stress, $\sigma_{i j}$, and the hydraulic pressure, $p$, and takes the following form:

$$
\sigma_{i j}^{\prime}=\sigma_{i j}-p \delta_{i j},
$$

where $\delta_{i j}$ is the Kronecker delta function.

When the direction of the stratified structural planes deviated from the coordinate system, as shown in Figure 1, the constitutive equations will be transformed to another formation associated with the dip angle of the stratified structural planes. According to the coordinate transformation, the elastic matrix $[D]$ in equation (3) will be changed to $\left[D^{\prime}\right]$ :

$$
\begin{aligned}
{\left[D^{\prime}\right] } & =\left[L_{1}\right][D]\left[L_{1}\right]^{T}, \\
{\left[L_{1}\right] } & =\left[\begin{array}{ccc}
\cos ^{2} \theta & \sin ^{2} \theta & -2 \sin \theta \cos \theta \\
\sin ^{2} \theta & \cos ^{2} \theta & 2 \sin \theta \cos \theta \\
\sin \theta \cos \theta & -\sin \theta \cos \theta & \cos ^{2} \theta-\sin ^{2} \theta
\end{array}\right],
\end{aligned}
$$

where $\left[L_{1}\right]$ is the stress coordinate transformation matrix and $\theta$ is the directional angle of stratified structural planes measured from the $x$-axis.

\subsection{Governing Equations for Anisotropic Seepage Field.} Essentially, groundwater flow through the rock slope is a fracture seepage problem. A large number of engineering practices show that the flow velocity is generally low, and thus, the relationship between the flow velocity, $u_{i j}$, and the pressure gradient, $\nabla p$, can be described by the famous Darcy's law:

$$
u_{i j}=-K_{i j} \nabla(p-\rho g),
$$

where $K_{i j}$ is the permeability coefficient tensor associated with stress; $\rho$ is the water density; and $g$ is the gravitational acceleration.

The continuity equation of the seepage without sources or sink under steady state condition can be expressed as

$$
\nabla \cdot\left(\rho u_{i j}\right)=0 .
$$

The seepage property of rock fracture is highly dependent on the aperture of the fracture. In general, a large width of the aperture will lead to a high permeability of the fracture. Because the normal stress is the main factor that affects the aperture of the fracture, the permeability of the stratified rock mass is controlled by the normal stress on the structural planes. Therefore, based on Louis's equation [22], the permeability coefficient tensor associated with the normal stress can be expressed as

$$
K_{i j}=\left[\begin{array}{cc}
K_{10} e^{-\alpha \sigma_{22}^{\prime}} & 0 \\
0 & K_{20} e^{-\alpha \sigma_{11}^{\prime}}
\end{array}\right],
$$

where $K_{10}$ and $K_{20}$ are the initial permeability coefficients in the principal directions of the stratified rock mass; $\sigma_{22}^{\prime}$ and $\sigma_{11}^{\prime}$ are the normal stresses in the directions of the corresponding initial permeability, respectively; and $\alpha$ is the coupled coefficient which can be determined by laboratory seepage tests. In this study, $\alpha$ is set to 0.1 with reference to Tang et al. [23].

The equation of seepage coupled with stress for plane strain condition is expressed in equation (8). When the coordinate system is noncoincident with the principal directions, the permeability coefficient tensor, $K_{i j}$, will be transformed into $K_{i j}^{\prime}$ according to the coordinate transformation:

$$
K_{i j}^{\prime}=\left[L_{2}\right] K_{i j}\left[L_{2}\right]^{T},
$$

where $\left[L_{2}\right]$ is the coordinates transformation matrix for the permeability coefficient, which reads as

$$
\left[L_{2}\right]=\left[\begin{array}{cc}
\cos \theta & \sin \theta \\
-\sin \theta & \cos \theta
\end{array}\right] .
$$

The numerical model is built by considering the anisotropy of the stress and seepage properties of the stratified rock slope. In the next section, the model will be numerically analyzed by the finite element software COMSOL because of the difficulty in analytical solution.

\section{Validation of the Model}

In this section, the numerical model will be validated by simulating the stress and seepage fields of an idealized slope. The simulation domain is $1800 \mathrm{~m} \times 800 \mathrm{~m}$, as shown in Figure 2. It is a symmetric slope with a height of $300 \mathrm{~m}$ and a dip angle of $45^{\circ}$. It is assumed that the rock mass is fixed at the bottom and free at the top with rollers boundaries on its left and right sides (allowing deformation only in the vertical direction). In addition, the gravity is applied as the body load. For the seepage analysis, both of the left and right seepage boundaries are set as the fixed water head of $800 \mathrm{~m}$, and the bottom seepage boundary is impervious, while the bottom of the slope boundary is set as the fixed water head of $550 \mathrm{~m}$. The 


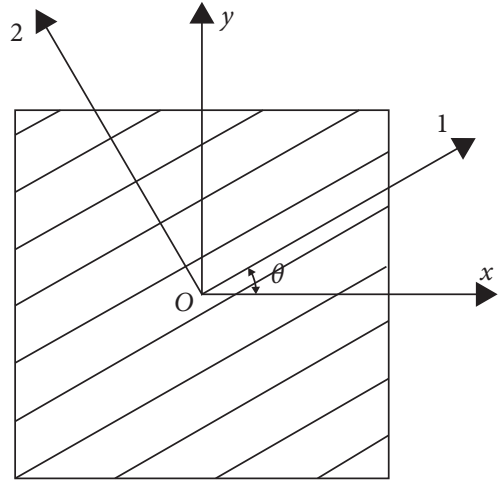

Figure 1: The relationship between stratified structural plane direction and the global coordinate system.

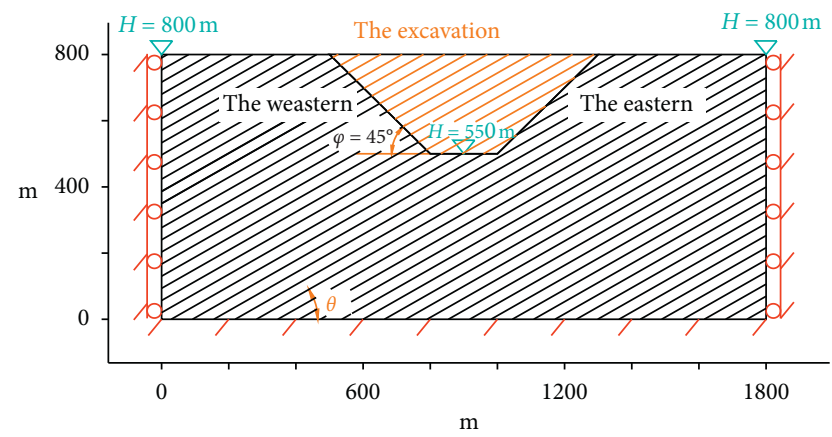

Figure 2: Numerical model of an idealized stratified rock slope used to validate the model.

physical and mechanical parameters of the rock mass are employed according to Zhang [24], as listed in Table 1.

Figure 3 gives the major principal stress vector of the slope under different directions of the stratified structural planes. The stress field shows anisotropy associated with the direction of the stratified structural planes. The orientation of the major principal stress is parallel to the profile in the vicinity of the slope, while it is parallel to the stratified structural plane direction in far field of the slope.

The rock stratum is in a state of static equilibrium and has stored elastic potential energy before the excavation. Once the excavation was finished, the elastic potential energy would be released which leads to the spring back of elastic deformation. Figure 4 shows the displacements of the slope under different directions of the stratified structural planes. It can be seen that the deformation field is significantly anisotropic. The spring back tends to occur vertically to the surface of stratified structural planes, i.e., the deformation of the slope in the normal direction of the surface of stratified structural planes is larger than that in other directions. This generally shows good agreement with the conclusion of the previous study [25].

Figure 5 shows the seepage fields and water tables of the slope under different directions of the stratified structural planes. In this figure, different colors are used to donate the different water heads. The seepage field is also affected significantly by the stratified structural planes. When the direction is horizontal, as shown in Figure 5(a), groundwater flows out of the slope surface more easily than infiltrating down. The water table is high. When the direction is vertical, as shown in Figure 5(d), the underground water flowing out of the slope surface is difficult but infiltrate down easily. Consequently, the water table is lower than that with horizontal stratified structural planes. The water table simulated and associated with the direction of the stratified structural plane compares well with that simulated by BEM [26].

Figure 6 shows the normal effective stress and principal permeability at the survey line $\mathrm{MN}$, as shown in Figure 5(b), where the upper extreme point, $\mathrm{M}$, is on the line of slope top and the lower extreme point, $\mathrm{N}$, is on the bottom line of the model. The line has a $100 \mathrm{~m}$ horizontal distance from the slope shoulder of the right slope. It can be seen that the hydraulic conductivity is constant without considering the coupling effect. When the stress-dependent permeability as given in equation (9) is considered, the permeability will decline with the increase in normal stress. It is because the compression effect inevitably leads to the closure of structural planes. In this regard, as it goes deeper, the normal stress increases, which leads to the decrease of the hydraulic conductivities in two major directions. The space variation of anisotropic permeability simulated with this model compares well with the field measurement [27]. Thus, it is reasonable to conclude that the proposed model is effective in capturing the mechanics of stratified rock slope.

\section{A Case Study}

4.1. Engineering Geology. The Fushun west open-pit mine located in Fushun, Liaoning, China, is chosen as the study area. The mine is a large-scale open-pit coal mine with a maximum length about $6.6 \mathrm{~km}$ in east-west direction and a maximum width about $2.2 \mathrm{~km}$ in north-south direction. The depth is about $400 \mathrm{~m}$ with an elevation from $+110 \mathrm{~m}$ to $-309 \mathrm{~m}$. The bottom of the open pit is the lowest elevation of all open pit mines in China. Its mining history is approximately 100 years. The southern slope of the open pit is a gentle slope with a slope angle varying between $25^{\circ}$ and $30^{\circ}$. The southern slope is mainly consisted of two rock strata, basalt in the shallow, and granitic gneiss in the deep. A set of stratified structural planes, with a spacing varying from $20 \mathrm{~cm}$ to $50 \mathrm{~cm}$, can be observed clearly in the shallow basalt stratum by the field and core observation [10]. The trend of the stratified structural planes distributes uniformly and the average dip angle is about $30^{\circ}$. The stratified structural planes with good connectivity in the trending direction are beneficial for groundwater flow. Most of the stratified structural planes in the boring-core have been corroded into light yellow which means they are the main channels for groundwater seepage. The fracture of the deep granitic gneiss stratum is undeveloped and its structure is comparatively complete comparing with the shallow rock stratum.

4.2. Numerical Model. According to the geologic conditions from the field geological survey, a numerical model for the numerical simulations of the cross-section E800 of the southern slope is established as a plain strain problem. The 
TABLE 1: Deformation parameters of slope rock mass.

\begin{tabular}{llcccccc}
\hline \multicolumn{2}{c}{$\begin{array}{c}\text { Young's modulus } \\
(\mathrm{GPa})\end{array}$} & & Poisson's ratio & \multicolumn{2}{c}{$\begin{array}{c}\text { Primary } \\
\text { permeability } \\
\left(\times 10^{-7} \mathrm{~m} / \mathrm{s}\right)\end{array}$} & $K_{2}$ & Shear modulus (GPa) \\
$E_{1}$ & $E_{2}$ & $v_{13}$ & $v_{12}$ & $v_{32}$ & $K_{1} /$ & $G_{13}$ \\
\hline 18.5 & 9.5 & 0.33 & 0.3 & 0.35 & 3.21 & 0.33 & 11.7 \\
\hline
\end{tabular}

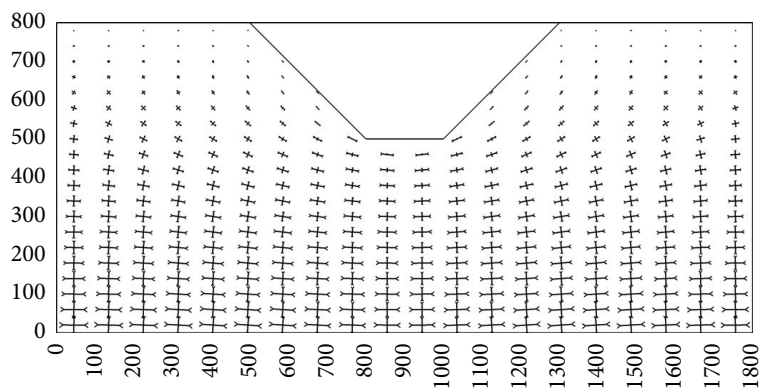

(a)

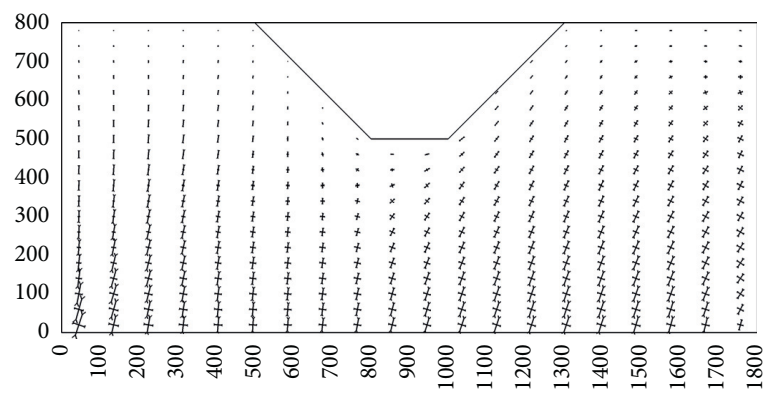

(c)

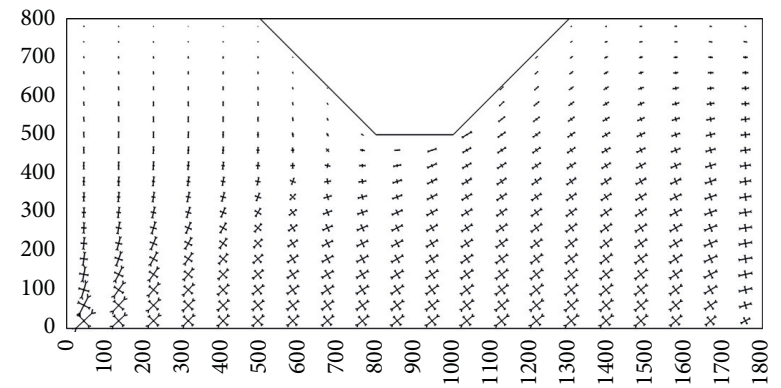

(b)

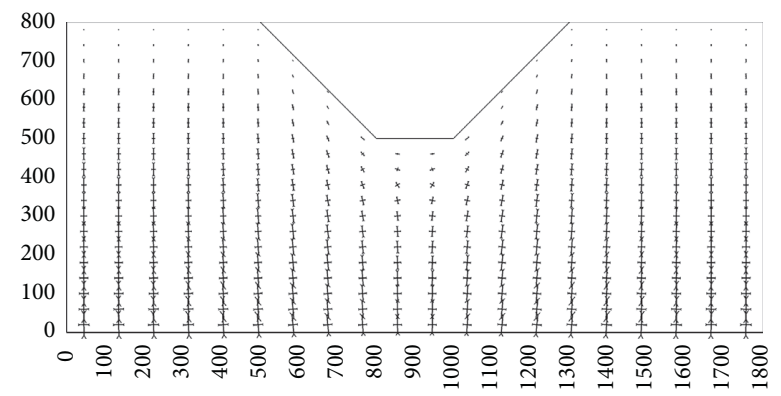

(d)

FIGURE 3: Principal stress vector of rock slope under different angles of stratified structural plane: (a) $\theta=0^{\circ}$, (b) $\theta=30^{\circ}$, (c) $\theta=60^{\circ}$, and (d) $\theta=90^{\circ}$.

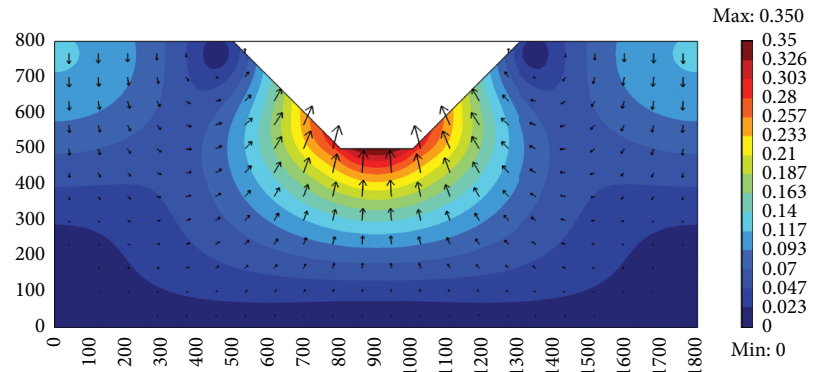

(a)

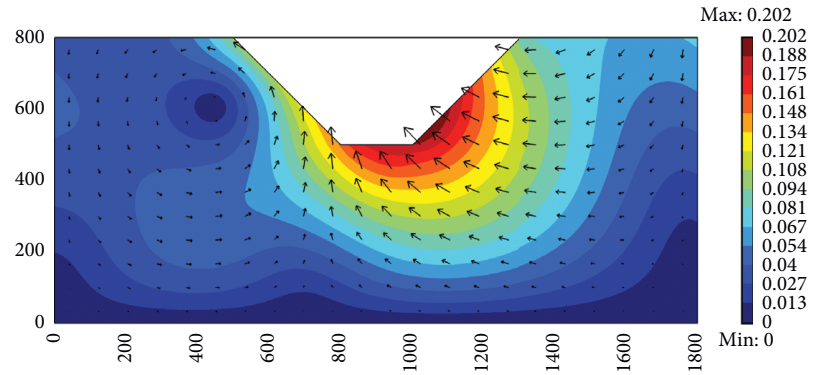

(c)

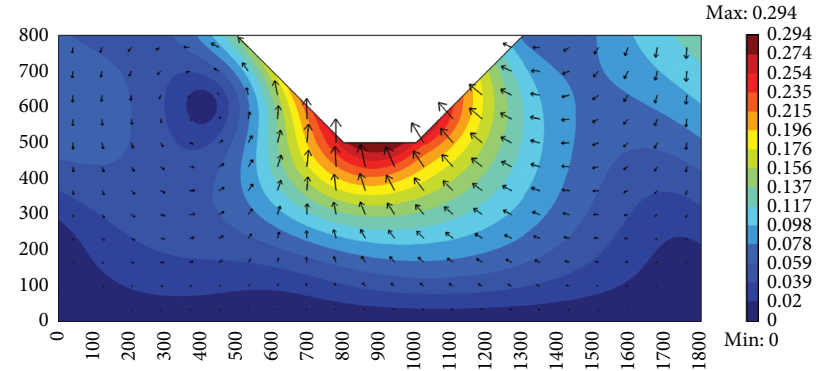

(b)

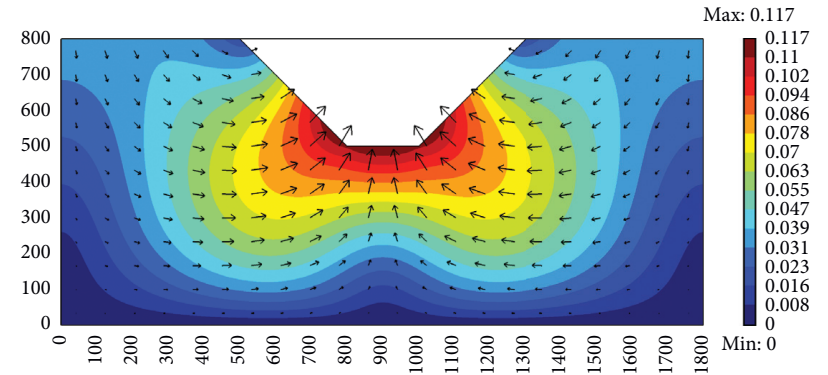

(d)

Figure 4: Displacement of rock slope under different angles of stratified structural plane: (a) $\theta=0^{\circ}$, (b) $\theta=30^{\circ}$, (c) $\theta=60^{\circ}$, and (d) $\theta=90^{\circ}$. 


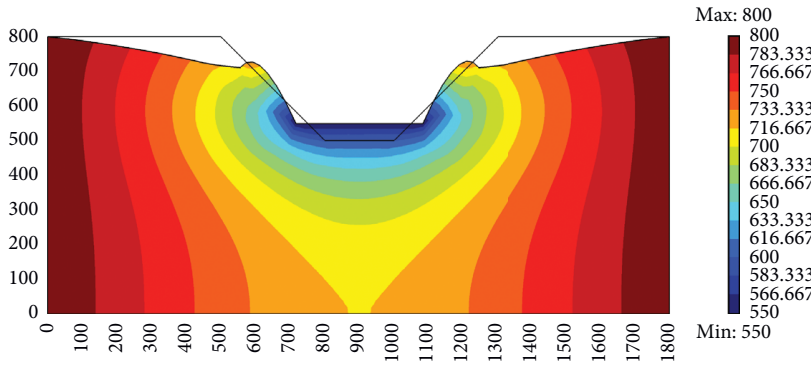

(a)

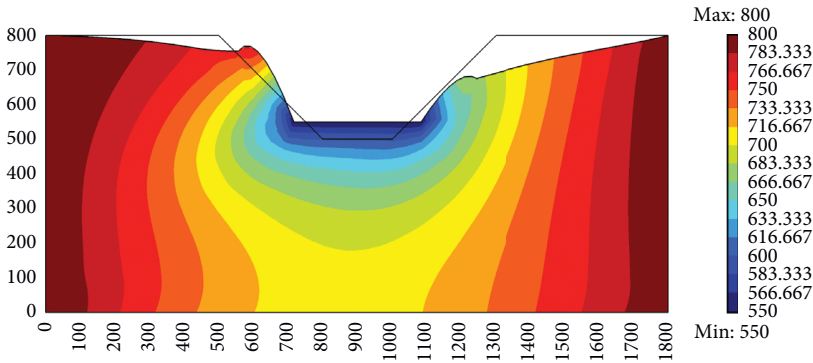

(c)

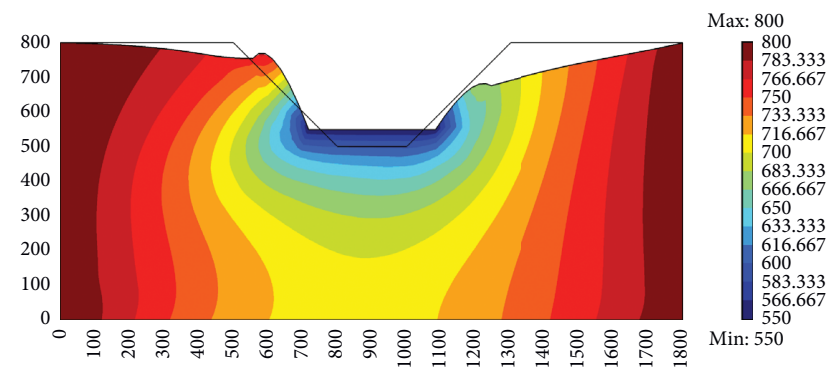

(b)

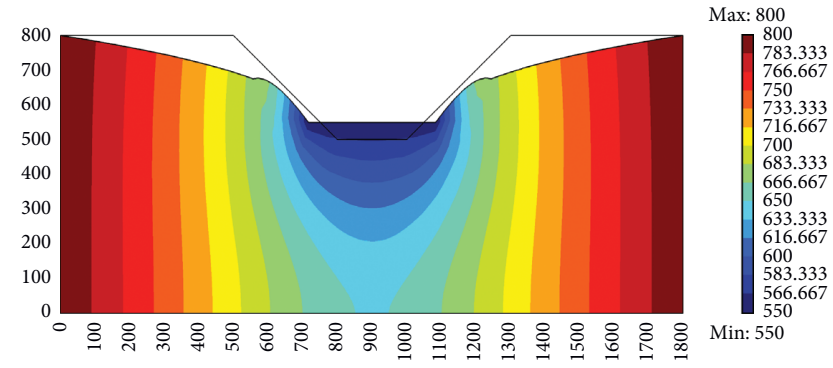

(d)

FIGURE 5: Seepage field of rock slope under different angles of stratified structural plane: (a) $\theta=0^{\circ}$, (b) $\theta=30^{\circ}$, (c) $\theta=60^{\circ}$, and (d) $\theta=90^{\circ}$.

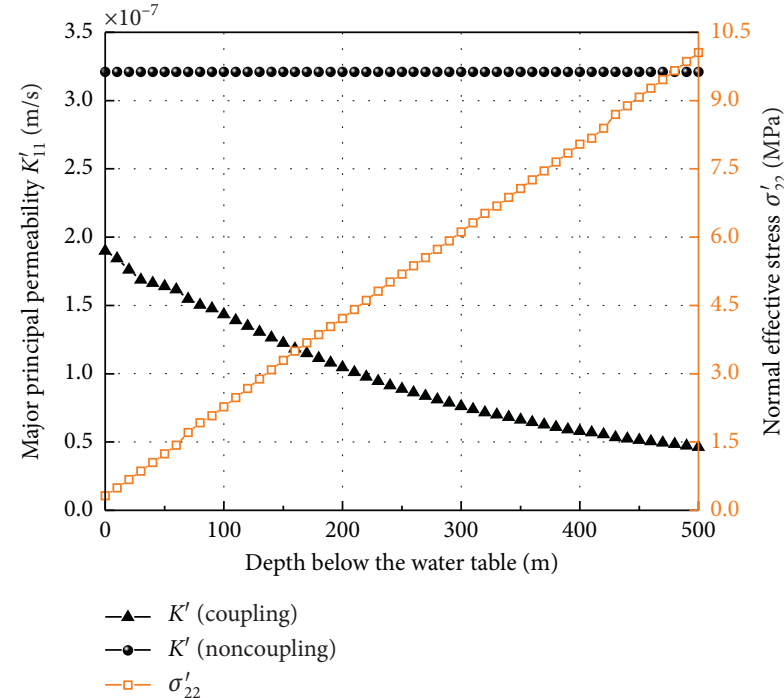

(a)

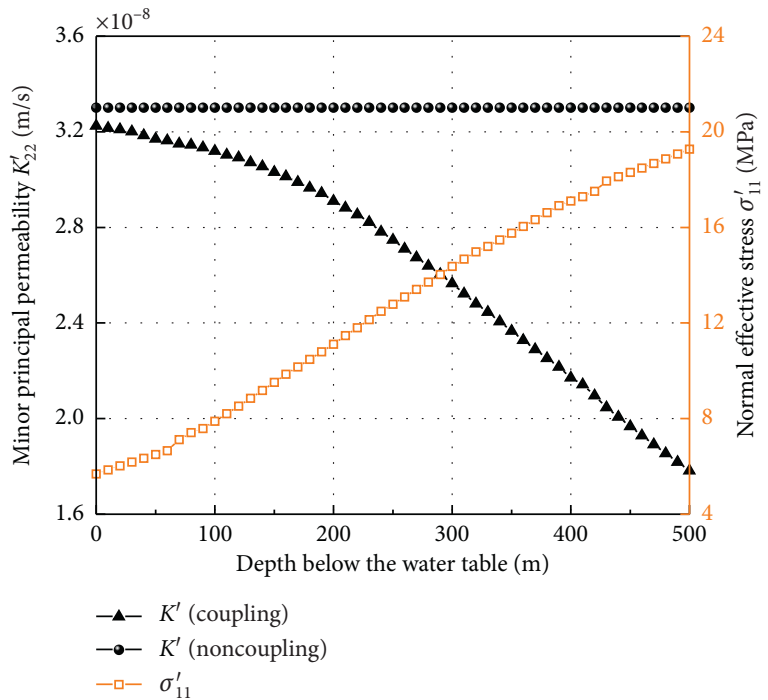

(b)

Figure 6: Normal effective stress and principal permeability at the survey line MN; (a) major principal permeability and (b) minor principal permeability.

geometry of the model together with the boundary conditions is illustrated in Figure 7. As shown in the figure, the numerical model is built with the shallow rock stratum and the deep rock stratum. The shallow rock stratum is the stratified basalt which is assumed to be anisotropic, and the deep one is the intact granitic gneiss which is assumed to be isotropic. There are two types of boundary conditions of the model: solid boundary conditions for stress analysis and hydraulic boundary conditions for seepage analysis. All the outer boundaries of the model except the slope surface are fixed in the normal direction, which can be expressed mathematically as $U_{\mathrm{n}}=0$. The water heads of $350 \mathrm{~m}$ and $700 \mathrm{~m}$ are specified at left and right boundaries, respectively. While no-flow condition is set on the bottom boundary of the model. Gravity is considered as the body force.

In order to fully comprehend the anisotropy and coupling property on the seepage, three cases are devised in the simulations, as listed in Table 2. For case I, the stratified basalt is calculated by the anisotropic seepage model, and the granitic gneiss is calculated by the isotropic seepage model. 


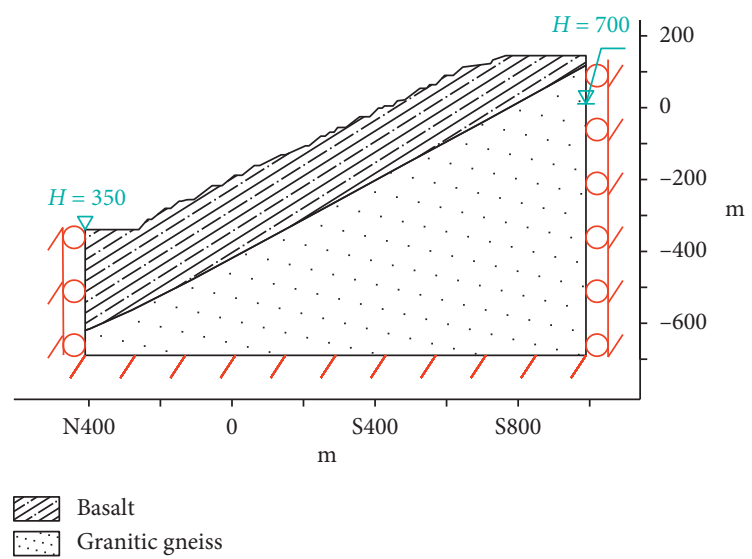

Figure 7: Numerical model of cross-section E800.

TABLE 2: Three cases considered in the numerical simulations.

Case
$\begin{aligned} & \text { I } \\ & \text { II }\end{aligned}$
III The anisotropic seepage model for the stratified basalt and the isotropic seepage model for the granitic gneiss
The typical seepage-stress coupled model for both the stratified basalt and the granitic gneiss
gneiss

TABle 3: Physicomechanical parameters of rock strata employed for the simulations.

\begin{tabular}{|c|c|c|c|c|c|c|c|c|c|}
\hline \multirow[t]{2}{*}{ Rock strata } & \multirow[t]{2}{*}{ Isotropy/anisotropy } & \multirow[t]{2}{*}{ Density $\left(\mathrm{kg} / \mathrm{m}^{3}\right)$} & \multicolumn{2}{|c|}{$\begin{array}{l}\text { Permeability } \\
\left(\times 10^{-6} \mathrm{~m} / \mathrm{s}\right)\end{array}$} & \multicolumn{2}{|c|}{$\begin{array}{c}\text { Young's } \\
\text { modulus } \\
(\mathrm{GPa})\end{array}$} & \multicolumn{2}{|c|}{$\begin{array}{l}\text { Poisson's } \\
\text { ratio }\end{array}$} & \multirow[t]{2}{*}{ Shear modulus (GPa) } \\
\hline & & & $K_{1}$ & $K_{2}$ & $E_{1}$ & $E_{2}$ & $v_{1}$ & $v_{2}$ & \\
\hline \multirow{2}{*}{ Basalt } & Anisotropy & 2800 & 1.95 & 0.64 & 26.9 & 14.5 & 0.23 & 0.3 & 7.25 \\
\hline & Isotropy & 2800 & \multicolumn{2}{|c|}{1.55} & \multicolumn{2}{|c|}{22.8} & \multicolumn{2}{|c|}{0.28} & 8.91 \\
\hline Granitic gneiss & Isotropy & 2540 & \multicolumn{2}{|c|}{1.05} & \multicolumn{2}{|c|}{45} & \multicolumn{2}{|c|}{0.22} & 18.44 \\
\hline
\end{tabular}

For case II, both the stratified basalt and the granitic gneiss are calculated by the typical seepage-stress coupled model. For case III, only the granitic gneiss is calculated by the typical seepage-stress coupled model, while the stratified basalt is calculated by the anisotropic seepage-stress coupled model. The solid mechanical and seepage parameters of the rock mass are listed in Table 3.

4.3. Numerical Results. Figure 8 shows the distribution of hydraulic head and flow velocity vector. It can be seen from the flow velocity field that the permeability of the shallow rock mass is high due to the good connectivity of the stratified structural planes, which have a good contribution to the formation of seepage channels. The groundwater flows toward the slope surface easily because the dominant orientation of the stratified structural planes is the same with the slope angle. As for case I, when the effect of stress on seepage is not considered, the permeability of the rock stratum is constant. Thus, the seepage shows homogeneity of each stratum. By contrast, for case II and III, because the stress is coupled on the seepage, the permeability as well as the velocity will decrease with the increase in the stratum depth. However, as for case II, due to the typical seepage- stress coupled model used in the simulation, the effect of the stratified structural planes on the seepage cannot be reflected. In this regard, the anisotropic seepage-stress coupled model is more capable to model the stratified basalt. In addition, the distribution of computational water table of each case and the actual measurement are shown in Figure 8(d). Obviously, the computational water table of case III compares better with the actual measurement.

Besides, the minor principal stress of case III is presented in Figure 9, in which the tensile stress concentration near the slope surface is indicated. Under the condition of tensile stress, the stratified structural planes of basalt are expanded, which is more helpful to the permeability enhancement and groundwater flow. Therefore, the water table calculated with stress coupled is lower than that without stress coupled.

The minor principal stress for case III presented in Figure 9 shows that some tensile stress concentrations occur near the slope surface. Because rock mass usually has the properties of low tensile strength, the rock mass near the slope surface may be unstable. However, shear failure is a more common failure mode for dip stratified rock slope compared with tensile failure $[1,10]$. If the cohesion and frictional angle of the basalt rock mass are set to $c=0.24 \mathrm{MPa}$ and $\varphi=29^{\circ}$, respectively, and $c=1.2 \mathrm{MPa}, \varphi=40^{\circ}$ for the 


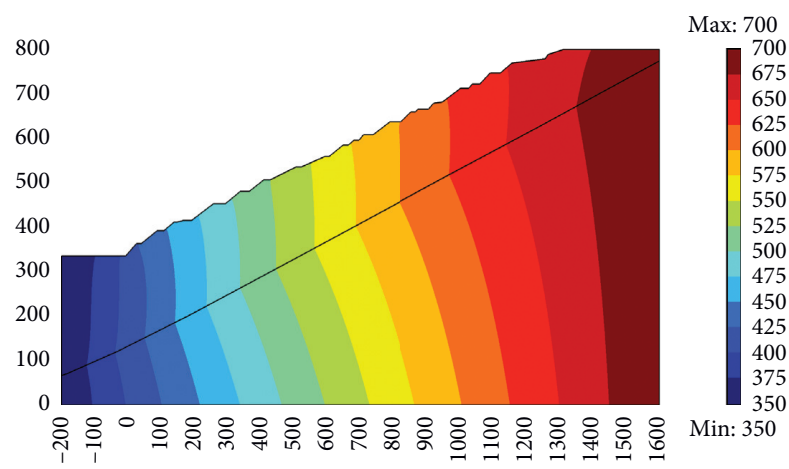

(a)

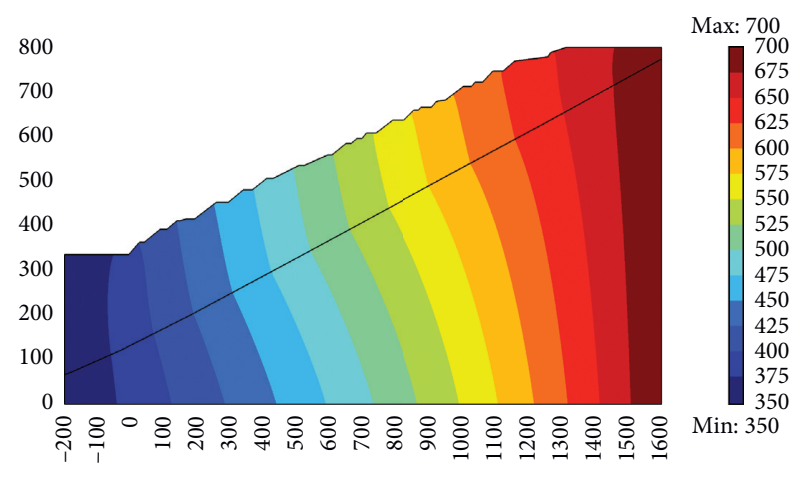

(c)

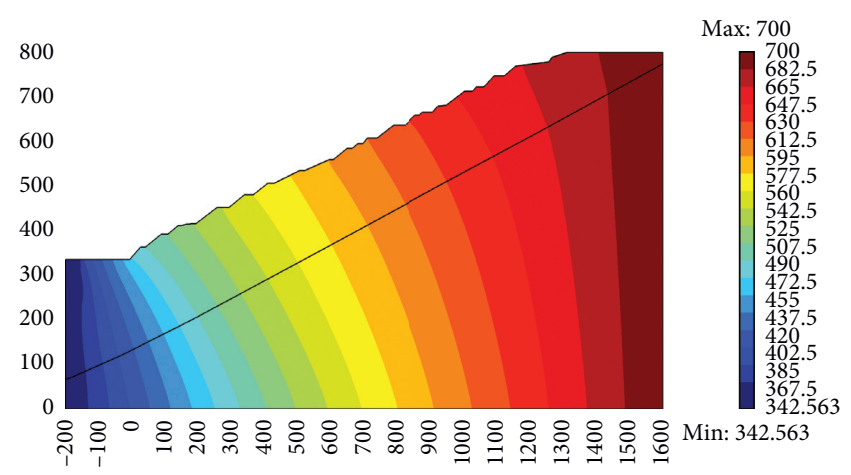

(b)

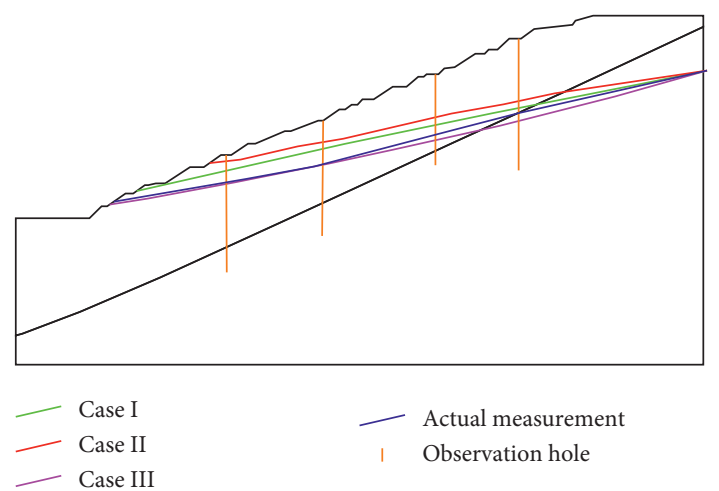

(d)

Figure 8: Distribution of hydraulic head and flow velocity vector; (a) case I, (b) case II, (c) case III, and (d) water table.

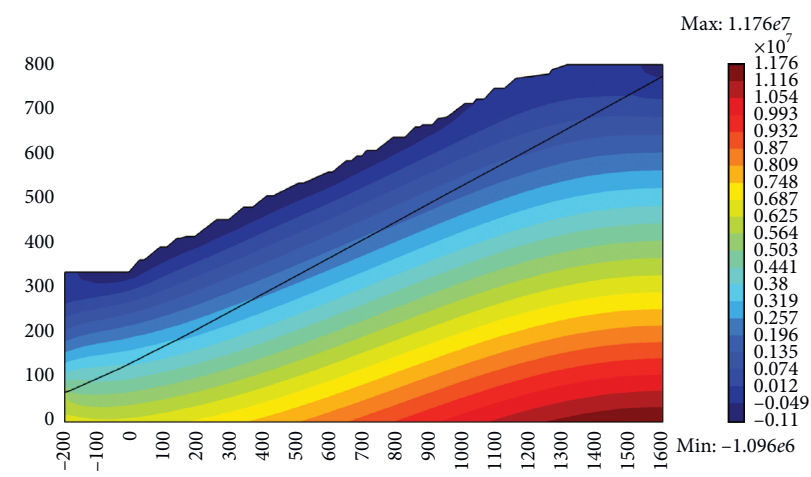

Figure 9: Minor principal stress of the slope for case III.

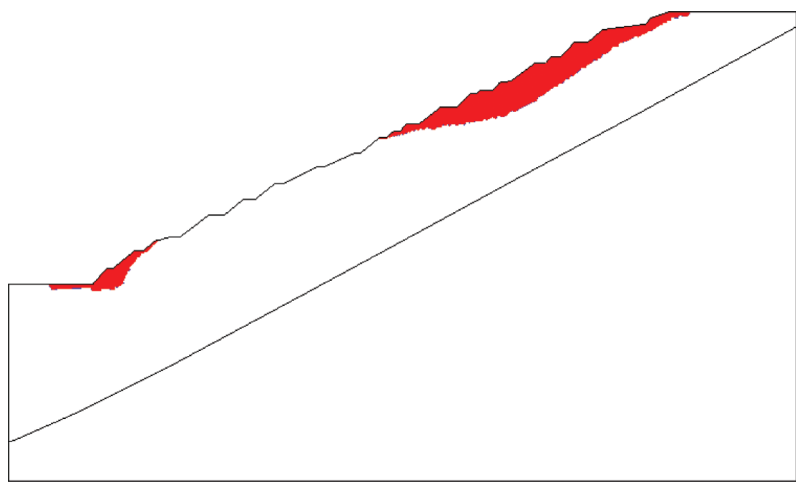

Figure 10: Shear damage zone of the slope for case III. 
granitic gneiss according to Yang et al. [10]; the shear damage zone of the slope can be obtained by Molar-Coulomb strength criterion, as shown in Figure 10. Two damage zones can be found in the shallow basalt rock mass: one distributes at the upper part of the slope surface and the other distributes at the slope toe. The two damage zones are not connected, which means that the local part of the slope rock mass may be unstable, but the whole slope is stable.

\section{Conclusion}

The anisotropy is a significant characteristic of stratified rock mass due to the stratified structural planes. For slope engineering, it is of vital importance to understand the anisotropic seepage and stress characteristics of the stratified rock slope in order to evaluate scientifically the stability of stratified rock slope and optimize slope angle. In this work, an anisotropic seepage-stress coupled model is proposed to simulate the stress and seepage fields of the stratified rock slope. The conclusions can be drawn as follows:

(1) This model is validated by simulating the stress and seepage fields of an idealized slope; the deformation and stress-dependent permeability of the slope is predicted and compared well with the previous study. It indicates the proposed model is effective in characterizing the mechanics behavior of rock slope that is dominantly affected by the rock mass anisotropy.

(2) The groundwater seepage and deformation of rock slope are strongly dependent on the stratified structural planes. When the slope is excavated in the horizontal stratified rock strata, a floor heave deformation will be observed in the bottom of the slope due to the unloading effect. For dip stratified rock slope, the deformation is more likely to be observed near the slope toe. For the same stratum, the deformation of dip stratified rock slope is more significant than that of the counter-tilt slope.

(3) Dip stratified rock structure is more beneficial for groundwater seepage than counter-tilt rock structure. Therefore, the water table of dip stratified rock slope is generally lower than the counter-tilt rock slope.

(4) The permeability of the stratified basalt of the southern slope at Fushun west open-pit mine is strongly anisotropic, and the results simulated by using the proposed anisotropic analysis are in good agreement with the actual measurement.

\section{Data Availability}

The data used to support the findings of this study are included within the article.

\section{Conflicts of Interest}

The authors declare that they have no conflicts of interest.

\section{Acknowledgments}

This research was financially supported by the National Key Research and Development Program of China (2017YFC1503100 and 2016YFC0801602), the Natural Science Foundation of China (U1903216 and 51909032), a project funded by the Priority Academic Program Development of Jiangsu Higher Education Institutions, the Youth fund of Suzhou University of Science and Technology (XKQ2018005), and the Science and Technology Project of Jiangsu Construction System (2018ZD033).

\section{References}

[1] J. Franz, An Investigation of Combined Failure Mechanisms in Large Scale Open Pit Slopes, The University of New South Wales, Sydney, Australia, 2009.

[2] L. Pantelidis, "Rock slope stability assessment through rock mass classification systems," International Journal of Rock Mechanics and Mining Sciences, vol. 46, no. 2, pp. 315-325, 2009.

[3] R. Chowdhury and B. N. Rao, "Probabilistic stability assessment of slopes using high dimensional model representation," Computers and Geotechnics, vol. 37, no. 7-8, pp. 876-884, 2010.

[4] A. Johari, A. Fazeli, and A. A. Javadi, "An investigation into application of jointly distributed random variables method in reliability assessment of rock slope stability," Computers and Geotechnics, vol. 47, no. 47, pp. 42-47, 2013.

[5] J. M. Duncan, "State of the art: limit equilibrium and finiteelement analysis of slopes," Journal of Geotechnical Engineering, vol. 123, no. 9, pp. 577-596, 1996.

[6] R. Baker, "Determination of the critical slip surface in slope stability computations," International Journal for Numerical \& Analytical Methods in Geomechanics, vol. 4, no. 4, pp. 333-359, 2010.

[7] K. Ma, C. A. Tang, L. C. Li, P. G. Ranjith, M. Cai, and N. W. Xu, "3D modeling of stratified and irregularly jointed rock slope and its progressive failure," Arabian Journal of Geosciences, vol. 6, no. 6, pp. 2147-2163, 2013.

[8] J. F. Zhang, Z. G. Li, and T. Qi, "Mechanism analysis of landslide of a layered slope induced by drawdown of water level," Science in China, vol. 48, no. 1, pp. 136-145, 2005.

[9] D. V. Griffiths and P. A. Lane, "Slope stability analysis by finite elements," Geotechnique, vol. 49, no. 7, pp. 653-654, 1999.

[10] T.-H. Yang, W.-H. Shi, P.-T. Wang, H.-L. Liu, Q.-L. Yu, and Y. Li, "Numerical simulation on slope stability analysis considering anisotropic properties of layered fractured rocks: a case study," Arabian Journal of Geosciences, vol. 8, no. 8, pp. 1-9, 2015.

[11] G. Shi, Discontinuous Deformation Analysis: A New Numerical Model for the Statics and Dynamics of Block Systems, University of California, Berkeley, CA, USA, 1988.

[12] H. Liu, W. Zhu, Y. Yu, T. Xu, R. Li, and X. Liu, "Effect of water imbibition on uniaxial compression strength of sandstone," International Journal of Rock Mechanics and Mining Sciences, vol. 127, Article ID 104200, 2020.

[13] Y. Zhang, "Research status of mathematical model of seepage," Yangtze River, vol. 22, no. 3, pp. 1-10, 1991.

[14] B. Amadei, "Importance of anisotropy when estimating and measuring in situ stresses in rock," International Journal of Rock Mechanics and Mining Sciences \& Geomechanics Abstracts, vol. 33, no. 3, pp. 293-325, 1996. 
[15] A. F. Fossum, "Effective elastic properties for a randomly jointed rock mass," International Journal of Rock Mechanics and Mining Sciences \& Geomechanics Abstracts, vol. 22, no. 6, pp. 467-470, 1985.

[16] J.-W. Cho, H. Kim, S. Jeon, and K.-B. Min, "Deformation and strength anisotropy of asan gneiss, boryeong shale, and yeoncheon schist," International Journal of Rock Mechanics and Mining Sciences, vol. 50, no. 2, pp. 158-169, 2012.

[17] M. Hakala, H. Kuula, and J. A. Hudson, "Estimating the transversely isotropic elastic intact rock properties for in situ stress measurement data reduction: a case study of the Olkiluoto mica gneiss, Finland," International Journal of Rock Mechanics and Mining Sciences, vol. 44, no. 1, pp. 14-46, 2007.

[18] C. D. Wang, C. S. Tzeng, E. Pan, and J. J. Liao, "Displacements and stresses due to a vertical point load in an inhomogeneous transversely isotropic half-space," International Journal of Rock Mechanics and Mining Sciences, vol. 40, no. 5, pp. 667-685, 2003.

[19] R. A. Everitt and E. Z. Lajtai, "The influence of rock fabric on excavation damage in the lac du bonnett granite," International Journal of Rock Mechanics and Mining Sciences, vol. 41, no. 8, pp. 1277-1303, 2004.

[20] I. Song, M. Suh, Y.-K. Woo, and T. Hao, "Determination of the elastic modulus set of foliated rocks from ultrasonic velocity measurements," Engineering Geology, vol. 72, no. 3-4, pp. 293-308, 2004.

[21] K. Terzaghi, R. B. Peck, and G. Mesri, "Soil mechanics in engineering practice,” Soil Science, vol. 68, no. 5, pp. 149-150, 2014.

[22] C. Louis, Rock Hydraulics, Springer, Vienna, Austria, 1972.

[23] C. A. Tang, L. G. Tham, P. K. K. Lee, T. H. Yang, and L. C. Li, "Coupled analysis of flow, stress and damage (FSD) in rock failure," International Journal of Rock Mechanics and Mining Sciences, vol. 39, no. 4, pp. 477-489, 2002.

[24] G. Zhang, Study on Equivalent Orthotropic Mechanical Parameters and Yield Criterion of Jointed Rock Mass and its Engineering Application, Hohai University, Nanjing, China, 2006.

[25] H. C. Zhu, A. Patrick, and H. Y. Zhong, "Numerical modelling methods and application for jointed rock mass, part 2: application in engineering practice," Chinese Journal of Rock Mechanics \& Engineering, vol. 24, no. 1, pp. 89-96, 2005.

[26] T. H. Yang and C. A. Tang, "Research into the permeation character of the opencast side-slope rock mass and the simulation of underground drainage," Journal of China Coal Society, vol. 24, no. 1, pp. 74-77, 1999.

[27] J. Sun and Z. Zhao, "Effects of anisotropic permeability of fractured rock masses on underground oil storage caverns," Tunnelling and Underground Space Technology, vol. 25, no. 5, pp. 629-637, 2010. 